\title{
Editor's Comment on COVID-19 and Psychiatry Education
}

\author{
Ann C. Schwartz ${ }^{1}$ \\ Accepted: 22 October 2020 / Published online: 29 October 2020 \\ (C) Academic Psychiatry 2020
}

The COVID-19 pandemic has dramatically impacted almost every sector of American society and has transformed our lives in a matter of months. It has altered how we practice psychiatry and teach our trainees. Amid this crisis, psychiatry educators and academic leaders have faced numerous challenges, including prioritizing trainee protection and education and ensuring high-quality treatment for patients. The selections from the online topical collection "COVID-19 and Psychiatry Education" [1] printed in the December 2020 issue of Academic Psychiatry offer two educational case reports and various perspectives from medical students, residents, fellows, and faculty that explore the impact of the pandemic on training and education as well as on well-being. They help provide insight into how this pandemic has uniquely affected psychiatric education.

During the initial weeks of the pandemic, training directors had to respond quickly to the crisis in education delivery, focusing on creating new workflows across clinical settings to minimize in-person exposure while preserving access and quality treatment for patients. While the pandemic catalyzed the rapid movement of health care to telehealth platforms and offered numerous benefits, it also created challenges. Those working onsite in inpatient and consultation-liaison settings worried about their own safety and the health of their family. Training directors had to consider how to best protect patients and staff and specifically how to enforce safe practices in an environment that includes patients with impaired thinking and judgment. Along with the massive clinical adjustments to attenuate viral spread, psychiatry training programs transitioned from in-person to remote teaching and supervision.

Psychiatry educators and academic leaders are faced with considering the immediate and long-term consequences of the COVID-19 pandemic on trainee safety and well-being. Perspectives written by trainees describe their struggles with

Ann C. Schwartz

aschwa2@emory.edu

1 Emory University School of Medicine, Atlanta, GA, USA the effects of ongoing restrictions to mitigate viral transmission with social distancing yet also highlight their resilience as they respond to the crisis and find ways to contribute meaningfully.

Uncertainty continues to linger, as we try to readjust to the multiple ways that our world has changed. As the pandemic evolves, we must continue to make timely modifications to the educational curriculum in preparation for the challenges ahead.

We encourage readers to submit papers with data about the impact of COVID-19 on education in psychiatry as well as educational interventions during the pandemic [2]. We are interested both in how programs have responded to the acute crisis and in how programs are teaching about the different intersections of COVID-19 and mental health. We look forward to reviewing your submissions.

\section{Compliance with Ethical Standards}

Conflict of Interest The author declares that she has no conflicts of interest.

\section{References}

1. Academic Psychiatry. COVID-19 and Psychiatry Education. Available at https://link.springer.com/journal/40596/ topicalCollection/AC_ab08ae134da8fbdf449373f19aec4656/page/1. Last accessed 21 October 2020.

2. Academic Psychiatry. Call for Papers: COVID-19 and Psychiatry Education. Available at https://www.springer.com/journal/40596/ updates/18088082. Last accessed 21 October 2020.

Publisher's Note Springer Nature remains neutral with regard to jurisdictional claims in published maps and institutional affiliations. 